\title{
Two-neutron radioactivity and four-nucleon emission from exotic nuclei
}

\author{
L. V. Grigorenko, ${ }^{1,2,3,4}$ I. G. Mukha, ${ }^{2}$ C. Scheidenberger, ${ }^{2,4}$ and M. V. Zhukov ${ }^{5}$ \\ ${ }^{1}$ Flerov Laboratory of Nuclear Reactions, JINR, RU-141980 Dubna, Russia \\ ${ }^{2}$ GSI Helmholtzzentrum für Schwerionenforschung, D-64291 Darmstadt, Germany \\ ${ }^{3}$ Russian Research Center "The Kurchatov Institute," RU-123182 Moscow, Russia \\ ${ }^{4} J u s t u s-L i e b i g-U n i v e r s i t a ̈ t ~ G i e s s e n, ~ D-35392$ Giessen, Germany \\ ${ }^{5}$ Fundamental Physics, Chalmers University of Technology, S-41296 Göteborg, Sweden
}

(Received 25 July 2011; published 29 August 2011)

\begin{abstract}
A theoretical method for estimates of a width of "true" four-nucleon decays is developed. Several candidate isotopes to possess this property are considered. Our lifetime estimates compare one-neutron, true two-neutron, and true four-neutron emissions at similar conditions. Prospects of experimental search for two-neutron, fourproton, and four-neutron radioactivity are discussed.
\end{abstract}

DOI: 10.1103/PhysRevC.84.021303

PACS number(s): 25.45.Hi, 24.50.+g, 24.70.+s, 27.20.+n

Nuclear disintegration via fission or the emission of protons, $\alpha$-particles, or nuclear clusters is a phenomenon that has been investigated since more than one century ago. This research has laid the foundation to the understanding of atomic nuclei, it exhibits many different facets of the strong force and has a large variety of applications. Nevertheless, neutron radioactivity, i.e., the spontaneous emission of one or more neutrons from the ground state of a nucleus, has not yet been observed experimentally. This will be a challenge for present and nextgeneration radioactive beam facilities. Even more surprising is the fact that there is no theoretical treatise of this phenomenon.

In this paper, we approach this question via two-proton $(2 p)$ radioactivity, which is the most-recently discovered mode of radioactive decay. Predicted by Goldansky [1] in 1960 , it was observed only 42 years later [2,3] in ${ }^{45} \mathrm{Fe}$. Now this process is under intense investigations, which provide new decay cases [4-6], detailed correlations of fragments [7], and new candidates for prospective studies $[8,9]$. The reason for the phenomenon of $2 p$ radioactivity (or $a$ "true" $2 p$ decay in the case of short-lived states) is founded in the specific energy conditions of daughter nuclei, which make one-proton emission energetically impossible; see Fig. 1(a). In this case, both protons have to be emitted simultaneously, which results in specific lifetime systematics (much longer lifetimes compared to ordinary binary decays) and complicated correlation patterns among the decay fragments. Such energy conditions evidently take place in systems with an even number of valence nucleons due to the pairing interaction.

There are two straightforward generalizations of $2 p$ radioactivity (true $2 p$ decay) phenomenon, which we discuss in this article in order to explore theoretically and experimentally two new decay modes:

(i) The direct analog of true $2 p$ decay across the isobar is the true two-neutron ( $2 n$ ) decay. With the progress in reaching experimentally the neutron drip-line, the interest to nuclei beyond this limit is increasing. E.g., some aspects of such processes were discussed by Thoennessen [10]. There is possibility that neutron(s) emission may take the form of $2 n$ radioactivity.

(ii) It is possible that two more nucleons added on top of a true two-nucleon precursor compose a system that has only a true four-nucleon decay branch; see Fig. 1(b). True four-nucleon decays are defined by energy conditions $S_{4 N}<0$ and $\left\{S_{N}, S_{2 N}, S_{3 N}\right\}>0$, in analogy with a true two-nucleon decay. This is an alternative to a sequential $(2 N)-(2 N)$ decay, which is illustrated in Fig. 1(c) with the example of ${ }^{8} \mathrm{C}$ decay [11]. As pointed out before (e.g., in Ref. [12]), the important feature of the true few-body emission is the existence of effective few-body "centrifugal barriers," which increase rapidly as the number of emitted particles increases. Therefore, the prospects to search four-neutron $(4 n)$ radioactivity could be more promising than the search for $2 n$ radioactivity. This will be discussed and compared in the following.

True four-nucleon emission was not investigated in reasonable detail before. The results of previous estimates [13] can be improved considerably, in particular in view of the meanwhile existing experience of the simplified approaches to $2 p$ radioactivity $[14,15]$. This calls for the development of corresponding theoretical methods, which will provide guidance toward new experiments.

Width estimates for true few-nucleon emission. The derivation of the expression for the decay width due to true four-nucleon emission in the proposed approximation is very similar to the derivation of the true two-nucleon width in Refs. [14,15]. So, here we only outline the major steps.

To derive an expression for the width we use the resonance wavefunction (WF) with pure outgoing asymptotic

$$
(H-\tilde{E}) \Psi^{(+)}=0
$$

and complex energy $\tilde{E}=E+i \Gamma / 2$. The major approximation is that a simplified Hamiltonian $H_{s}$ is introduced instead of the complete Hamiltonian $H$ for $k$ particles. In the three-body ( $2 N$ emission) case,

$$
\begin{aligned}
H & =T+V_{C N_{1}}+V_{C N_{2}}+V_{N_{1} N_{2}}, \\
H_{S} & =T+V_{C N_{1}}+V_{C N_{2}}+V_{3},
\end{aligned}
$$

where the potential $V_{3}$ is chosen to be localized in the region where all three particles are close to each other. In the five-body ( $4 \mathrm{~N}$ emission) case, the complete and simplified Hamiltonians are

$$
\begin{aligned}
H & =T+\sum_{i}^{4} V_{C N_{i}}+\sum_{i>j}^{4} V_{N_{i} N_{j}}, \\
H_{s} & =T+\sum_{i}^{4} V_{C N_{i}}+V_{5} .
\end{aligned}
$$



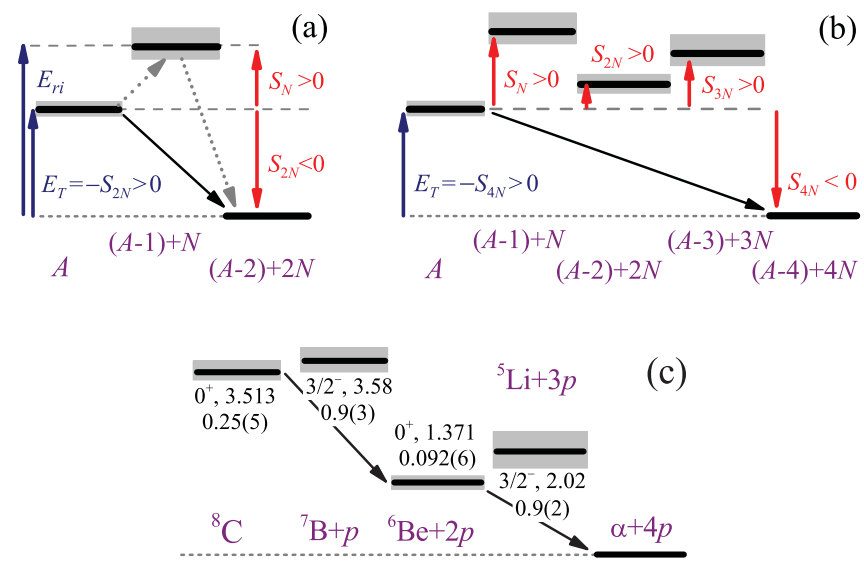

FIG. 1. (Color online) Energy conditions for various nuclear decay modes: (a) true two-nucleon, (b) true four-nucleon, (c) sequential $(2 N)-(2 N)$ emission with the example of ${ }^{8} \mathrm{C}$ decay. $S_{k N}$ is separation energy of $k$ nucleons. For the decay scheme of ${ }^{8} \mathrm{C}$ spin parities $J^{\pi}$, decay energies $E_{T}$, and widths $\Gamma$ (in $\mathrm{MeV}$ ) are indicated.

An auxiliary Hamiltonian $\bar{H}_{s}$ is defined for the simplified Hamiltonian. For example, in the three-body case it reads

$$
H_{s}=\bar{H}_{s}+V_{3}, \quad \bar{H}_{s}=T+V_{C N_{1}}+V_{C N_{2}} .
$$

In the limit of an infinitely heavy core, Greens's function $\bar{G}$ for the auxiliary Hamiltonian is known in a compact analytical form. This is Greens's function for $k$ independent (in the sense that their dependence is only via sharing the total energy) particles:

$$
\begin{aligned}
& \bar{G}_{E_{T}}^{(+)}\left(\mathbf{r}_{1}, \ldots, \mathbf{r}_{m} ; \mathbf{r}_{1}^{\prime}, \ldots, \mathbf{r}_{m}^{\prime}\right) \\
& =\frac{E_{T}^{m-1}}{m(\pi i)^{m-1}} \int_{-\infty}^{\infty} d \varepsilon_{1} \ldots d \varepsilon_{m-1} G_{E_{1}}^{(+)}\left(\mathbf{r}_{1} ; \mathbf{r}_{1}^{\prime}\right) \\
& \quad \ldots G_{E_{m}}^{(+)}\left(\mathbf{r}_{m} ; \mathbf{r}_{m}^{\prime}\right),
\end{aligned}
$$

where $\quad m=k-1, \quad E_{i}=\varepsilon_{i} E_{T} \quad$ for $\quad i<m$, and $E_{m}=\left(1-\sum_{i=1}^{m-1} \varepsilon_{i}\right) E_{T}$. The parameters $\varepsilon_{i}$ indicate which fraction of total energy is in the $i$-th subsystem.

Equation (1) can be rewritten in terms of Greens's function $\bar{G}^{(+)}$for the auxiliary Hamiltonian of $k$ particles (we are interested in the cases $k=3,5$ ):

$$
\Psi^{(+)}=\bar{G}^{(+)} V_{k} \Psi^{(+)} .
$$

It is conventional to introduce the WF

$$
\tilde{\Psi}^{(+)}=\Psi^{(+)}\left(\int_{V} d \mathbf{r}_{1} \ldots d \mathbf{r}_{m}\left|\Psi^{(+)}\right|^{2}\right)^{-1 / 2},
$$

normalized in the internal region $V$ with an enclosing surface $S$. Then the corresponding width $\Gamma$ (as the flux $j$ divided by the internal normalization $N$ ) is:

$$
\Gamma=j / N=\frac{\left.\left\langle\Psi^{(+)}|\hat{j}| \Psi^{(+)}\right\rangle\right|_{S}}{\left\langle\Psi^{(+)} \mid \Psi^{(+)}\right\rangle_{V}}=\left.\left\langle\tilde{\Psi}^{(+)}|\hat{j}| \tilde{\Psi}^{(+)}\right\rangle\right|_{S} .
$$

Assuming the localized character of the potential $V_{k}$, we replace the WF with outgoing asymptotic $\tilde{\Psi}^{(+)}$in Eq. (3) with the normalized quasi-stationary WF $\tilde{\Psi}$ obtained with "box" boundary conditions:

$$
\tilde{\Psi}^{(+)}=\bar{G}^{(+)} V_{k} \tilde{\Psi} .
$$

Using Eqs. (3) and (6) and the simplified Hamiltonian, the width for a $k$-body decay ( $m$-nucleon emission) is

$$
\Gamma=\frac{2^{3 m-1}}{m^{2} \pi^{m-1}} \int d \varepsilon_{i} \ldots d \varepsilon_{m-1} \frac{\left|A\left(\varepsilon_{1}, \ldots, \varepsilon_{m}\right)\right|^{2}}{v_{1} \ldots v_{m}},
$$

where $v_{i}=\sqrt{2 M_{i} E_{i}}$ is velocity of the $i$-th particle. After partial wave decomposition, the amplitude $A$ for a $k$-particle decay is obtained as

$$
\begin{aligned}
A\left(\varepsilon_{1}, \ldots, \varepsilon_{m}\right)= & \int d r_{1} \ldots d r_{m} \psi_{E_{1}, l_{1}}\left(r_{1}\right) \ldots \\
& \times \psi_{E_{m}, l_{m}}\left(r_{m}\right) V_{k} \tilde{\psi}\left(r_{1}, \ldots, r_{m}\right) .
\end{aligned}
$$

The functions $\psi_{E_{i}, l_{i}}$ are partial two-body continuum WFs normalized by the asymptotic condition

$$
\psi_{E, l}(r \rightarrow \infty)=e^{i \delta_{l}} \sin \left(k r+\delta_{l}\right) .
$$

In the quasi-stationary resonance approximation, these WFs can be approximated in the internal region for energies below and around the resonance as

$$
\psi_{E, l}(r)=\frac{1}{2} \frac{1}{E-E_{r}-i \Gamma(E) / 2} \sqrt{v \Gamma(E)} \tilde{\psi}_{E_{r}, l}(r),
$$

where $\tilde{\psi}_{E_{r}, l}$ is a quasi-stationary WF, defined on the resonance energy $E_{r}$ and normalized to unity in the internal region. The dependence of the width on energy is provided by the standard $R$-matrix expression

$$
\Gamma(E)=2 \gamma^{2} P_{l}\left(E, r_{\mathrm{ch}}, Z_{1}, Z_{2}\right),
$$

where $\gamma^{2}$ is the reduced width and $P_{l}$ is the penetrability, depending on energy, "channel radius" $r_{\mathrm{ch}}$, and charges $Z_{i}$. It was shown in Ref. [15] that the integral

$$
\left\langle V_{3}\right\rangle=\int d r_{1} d r_{2} \tilde{\psi}_{E_{1}, l_{1}}\left(r_{1}\right) \tilde{\psi}_{E_{2}, l_{2}}\left(r_{2}\right) V_{3} \tilde{\psi}\left(r_{1}, r_{2}\right)
$$

can be approximated as

$$
\left\langle V_{3}\right\rangle=D_{3}\left(E_{T}-E_{r_{1}}-E_{r_{2}}\right),
$$

where the coefficient $D_{3}$ is close to unity. In this approximation, the " $R$-matrix-like" expression for the widths can be obtained in the three-body case

$$
\begin{aligned}
\Gamma_{3}\left(E_{T}\right) \\
=\frac{E_{T}\left(E_{T}-E_{r_{1}}-E_{r_{2}}\right)^{2}}{2 \pi} \int_{0}^{1} d \varepsilon \frac{\Gamma_{r_{1}}\left(E_{1}\right)}{\left(E_{1}-E_{r_{1}}\right)^{2}+\Gamma_{r_{1}}^{2}\left(E_{1}\right) / 4} \\
\quad \times \frac{\Gamma_{r_{2}}\left(E_{2}\right)}{\left(E_{2}-E_{r_{2}}\right)^{2}+\Gamma_{r_{2}}^{2}\left(E_{2}\right) / 4} .
\end{aligned}
$$

The $E_{r_{i}}$ and $\Gamma_{i}$ are the energies and widths of the lowest resonance between the core and $i$-th nucleon. In the five-body case, the width is obtained in the same way:

$$
\begin{aligned}
\Gamma_{5}\left(E_{T}\right)= & \frac{E_{T}^{3}\left(E_{T}-\sum_{i=1}^{4} E_{r_{i}}\right)^{2}}{2 \pi^{3}} \int_{0}^{1} d \varepsilon_{1} \int_{0}^{1-\varepsilon_{1}} d \varepsilon_{2} \\
& \times \int_{0}^{1-\varepsilon_{1}-\varepsilon_{2}} d \varepsilon_{3} \prod_{i=1}^{4} \frac{\Gamma_{r_{i}}\left(E_{i}\right)}{\left(E_{i}-E_{r_{i}}\right)^{2}+\Gamma_{r_{i}}^{2}\left(E_{i}\right) / 4} .
\end{aligned}
$$



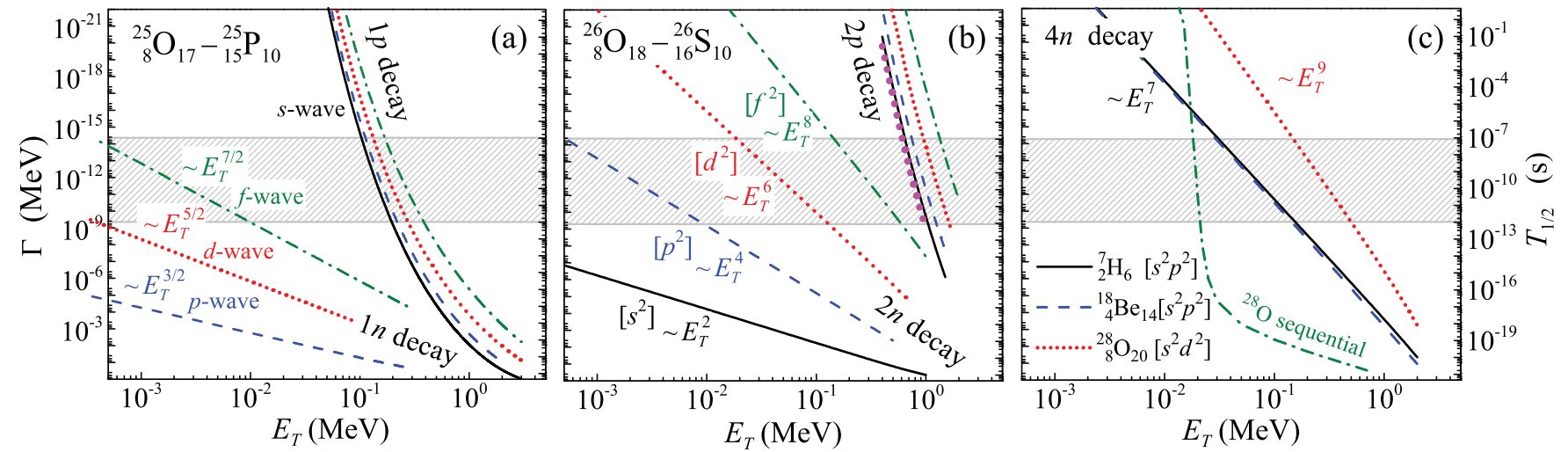

FIG. 2. (Color online) Estimated widths for (a) one-nucleon, (b) true two-nucleon, (c) true $4 n$ emission calculated for different orbital configurations. In the case of neutron emission, the low-energy behavior of the widths has an asymptotic dependence $\sim E_{T}^{\alpha}$, as is indicated next to the corresponding curves. The hatched area indicates the lifetime range accessible by decay-in-flight techniques. The results for ${ }^{26} \mathrm{~S}$ from Ref. [9] are represented in panel (b) by the thick dots. The dash-dotted curve in panel (c) provides the estimate of the sequential (2n)-( $2 n$ ) decay of ${ }^{28} \mathrm{O}$ via ${ }^{26} \mathrm{O}$ g.s., whose decay energy is $20 \mathrm{keV}$.

One- and two-neutron lifetime estimates. In the following, we will apply these findings to several cases of interest, which are depicted in Fig. 2. For the one-neutron emission, simple width estimates are obtained by the standard $R$-matrix expression Eq. (10) with the channel radius taken from systematics $r_{\mathrm{ch}}=1.4\left(A_{1}+A_{2}\right)^{1 / 3}$; see Fig. 2(a). Assuming a possible $s$ - or $d$-wave ground state (g.s.) for a nucleus with mass number $A=25$, one can see that the decay energy window for proton radioactivity, which can be detected with present detector technologies and thus corresponding to half-lives exceeding $\sim 1 \mathrm{ps}$, ranges from $\sim 50$ to $\sim 200 \mathrm{keV}$ for ${ }^{25} \mathrm{P}$. This range is narrow but not negligible: there is some probability for the g.s. to be found within this range. To identify neutron radioactivity in the isobar ${ }^{25} \mathrm{O}$, the decay energy $E_{T}$ should be smaller than $1 \mathrm{keV}$ even for the $d$-wave: it is very improbable that such a fine-tuned energy will actually be found. The realistic chance to find one-neutron radioactivity may appear only for $f$ wave and higher $l$ states. As the heavier neutron drip-line nuclei are not known, long-lived neutron emitters cannot be excluded.

For illustration, we consider theoretical estimates for the two pairs of isobaric partners ${ }_{16}^{26} \mathrm{~S}_{10}-{ }_{8}^{26} \mathrm{O}_{18}$ and ${ }_{15}^{25} \mathrm{P}_{10}-{ }_{8}^{25} \mathrm{O}_{17}$. These are not mirror nuclei, but these pairs have similar mass/charge ratios and should presumably have comparable structural and radial characteristics. ${ }^{26} \mathrm{~S}$ is a candidate to be a true $2 p$ precursor [9], and ${ }^{26} \mathrm{O}$ could be a long-living resonance and, thus, a true $2 n$ emitter [10]. Lifetime calculations for true two-nucleon decays are given in Fig. 2(b).

The possible $2 n$ radioactivity has a few important differences in comparison to the one-neutron radioactivity.

(i) Low-energy $s$-wave neutron emission could take place in the form of a virtual state, which cannot be interpreted in terms of width. For that reason, the neutron $s$-wave curve is missing in Fig. 2(a). In contrast, for the true $2 n$ emission the phase space for the three-body final state creates an additional effective centrifugal barrier. So, a narrow resonance state is formed even for the decay of a $\left[s^{2}\right]$ configuration. The possibility to observe narrow "three-body virtual states" built on $\left[s^{2}\right]$ configurations was discussed by Grigorenko and Zhukov [16] for ${ }^{10} \mathrm{He}$. The investigation of the $2 n$ ground-state decays has so far revealed only the existence of quite broad $(\Gamma \gtrsim 1 \mathrm{MeV})$ states in ${ }^{5} \mathrm{H}$, ${ }^{10} \mathrm{He}$, and ${ }^{13} \mathrm{Li}[13,17-19]$.

(ii) The decay-energy window for $2 p$ radioactivity of ${ }^{26} \mathrm{~S}$ ranges from 500 to $1700 \mathrm{keV}$, thus, is about 10 times broader than for the proton radioactivity of ${ }^{25} \mathrm{P}$. The energy window for the true $2 n$ decay is much broader than for one-neutron decay: for the $\left[d^{2}\right]$ and the $\left[f^{2}\right]$ configurations, the decays would be ascribed as radioactive for decay energies ranging up to 200 and $600 \mathrm{keV}$, respectively. Such energy ranges make the existence of true $2 n$ radioactivity much more probable.

(iii) At variance to the $1 n$ situation, the $2 n$ estimates in Fig. 2(b) should be interpreted as lifetime limits due to the possibility of configuration mixing. The $\left[s^{2}\right]$ and $\left[p^{2}\right]$ curves are likely to provide lower lifetime limits for $s-d$ and $p-f$ configurations, respectively. The $\left[d^{2}\right]$ and $\left[f^{2}\right]$ curves provide upper lifetime limits for them. From the experience collected in the $2 p$ decay studies, we may argue that the realistic results are located between the limiting curves but closer to that for the lower $l$ [4-6].

Four-neutron lifetime estimates. In Fig. 2(c), the estimates for true $4 n$ emission lifetimes are shown for ${ }^{7} \mathrm{H},{ }^{18} \mathrm{Be}$, and ${ }^{28} \mathrm{O}$. For simplicity, we used $E_{r_{i}}=1.5 \mathrm{MeV}$ in all cases. The other parameters are chosen according to guidelines from Ref. [15]. The orbital configurations were chosen to yield results closer to the upper limit for the width; the configurations with higher penetrability than $\left[s^{2} p^{2}\right]$ are not possible in the continuum of four neutrons. Therefore, the provided estimates can be considered as conservative. These estimates show that the trend of a decreasing width with increasing number of emitted particles is valid for a true $4 n$ emission as well. The true $4 n$ width is much smaller than the estimated $2 n$ width for the same energy. For illustration, the sequential decay estimate is provided in Fig. 2(c) for ${ }^{28} \mathrm{O}(2 n)-(2 n)$ decay via ${ }^{26} \mathrm{O}$ g.s. with $E_{T}=20 \mathrm{keV}$. Thus, long-lived states decaying by a true $4 n$ emission may be expected as radioactive precursors with 
decay energies up to hundreds of $\mathrm{keV}$, even for $p$ and $s-d$ shell nuclear configurations.

Energy conditions for true four-nucleon emission. In the above discussion, we considered only the lifetime dependences on energy without questioning whether the energy conditions needed for these estimates are realized in reality. At least in one known case $-{ }^{7} \mathrm{H}$ nucleus - one can assume that true $4 n$ decay takes place. The ground-state decay energies of the ${ }^{7} \mathrm{H}$ subsystems, ${ }^{4} \mathrm{H}[20]$ and ${ }^{5} \mathrm{H} \mathrm{[13]}$, are around $2 \mathrm{MeV}$. The information on ${ }^{6} \mathrm{H}$ is extremely poor, but the decay energy does not seem to be smaller than $2 \mathrm{MeV}$. The decay energy of ${ }^{7} \mathrm{H}$ is also uncertain but is likely to be around $1 \mathrm{MeV}$ and certainly smaller than $2 \mathrm{MeV}$ [21-23]. Thus, it is very likely that ${ }^{7} \mathrm{H}$ is a true ${ }^{4} \mathrm{n}$ emitter and as such it should have an anomalously small width, even for decay energies comparable to $1 \mathrm{MeV}$. For the lifetime of ${ }^{7} \mathrm{H}$ there is an upper limit of $1 \mathrm{~ns}$ [24]. This limit is based, however, on the production cross-section estimate, which we can find too optimistic nowadays [23]. Thus, an extreme long-lived g.s. is not excluded for this system.

The next candidates for true $4 n$ emission are ${ }^{18} \mathrm{Be}$ and ${ }^{28} \mathrm{O}$. We may separately discuss the energy conditions for ${ }^{28} \mathrm{O}$. The nucleus ${ }^{26} \mathrm{O}$ has been considered as candidate for $2 n$ radioactivity [25] and ${ }^{28} \mathrm{O}$ attracted sufficient attention due to its expected shell closure and, thus, the possibility that it is bound while the even-even precursor ${ }^{26} \mathrm{O}$ is unbound (e.g., Refs. [25,26]). The calculations have significant uncertainty (e.g., Ref. [26]) and this uncertainty is strongly increasing when we pass from ${ }^{26} \mathrm{O}$ to ${ }^{28} \mathrm{O}$. The predictions vary from practically bound ${ }^{28} \mathrm{O}$ to unbound by several $\mathrm{MeV}$. Thus, the energy conditions required for true $4 n$ emission are possibly fulfilled in ${ }^{28} \mathrm{O}$.

For proton-rich isotopes, it is difficult to expect that the energy conditions of true $4 p$ emission are fulfilled in reality. This statement is illustrated by the example of isobaric partners ${ }^{6} \mathrm{He}-{ }^{6} \mathrm{Be}$ and ${ }^{8} \mathrm{He}-{ }^{8} \mathrm{C}$. Their energies can be estimated in the independent particle model

$$
E_{6}{ }_{\mathrm{He}}=2 E_{5} \mathrm{He}+E_{\mathrm{nn}}\left({ }^{6} \mathrm{He}\right), \quad E_{8^{\mathrm{He}}}=2 E_{7^{7} \mathrm{He}}+E_{\mathrm{nn}}\left({ }^{8} \mathrm{He}\right) .
$$

Then, it can be found that the phenomenological pairing energy $E_{\mathrm{nn}}\left({ }^{6} \mathrm{He}\right) \approx 2.8 \mathrm{MeV}$ in ${ }^{6} \mathrm{He}$ is very close to $E_{\mathrm{nn}}\left({ }^{8} \mathrm{He}\right) \approx$ $3.1 \mathrm{MeV}$ in ${ }^{8} \mathrm{He}$ nucleus. Within the same estimates, the energies across the isobar are

$$
\begin{aligned}
E_{{ }^{6} \mathrm{Be}} & =2 E_{5^{H e}}+E_{\mathrm{nn}}\left({ }^{6} \mathrm{He}\right)+2 V_{\alpha-p}^{\text {coul }}+V_{p-p}^{\text {coul }}, \\
E_{{ }^{8} \mathrm{C}} & =2 E_{7^{7} \mathrm{He}}+E_{\mathrm{nn}}\left({ }^{8} \mathrm{He}\right)+4 V_{\alpha-p}^{\text {coul }}+6 V_{p-p}^{\text {coul }} .
\end{aligned}
$$

It is evident that Coulomb energy contributions are growing much faster than nuclear contributions with increasing number of valence nucleons. The energy conditions of true $2 p$ decay are realized in ${ }^{6} \mathrm{Be}$, but in ${ }^{8} \mathrm{C}$ the sequential $2 p-2 p$ emission [see Fig. 1(c)] is the dominating decay branch [11]. Therefore, at the neutron dripline, small variations of single-particle and pairing energies can easily lead to energy conditions of true $4 n$ emission. Beyond the proton dripline, some extreme situation should be realized to make true $4 p$ emission possible.

Prospects to explore $2 n$ and $4 n$ radioactivity. For experimental studies of true $2 n$ and $4 n$ decays of long-lived nuclear precursors, Fig. 2 can provide some first guidance. As typical decay energies will range from a few $\mathrm{keV}$ to a few

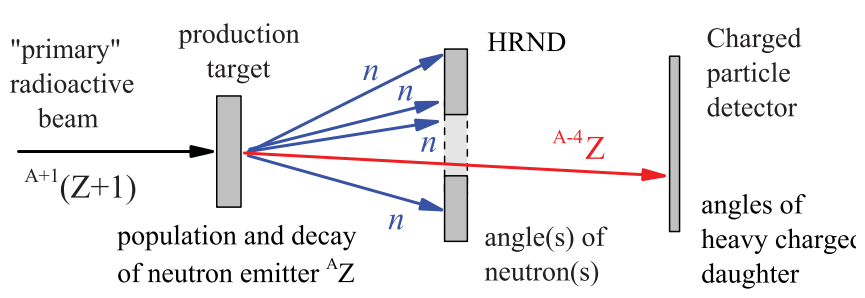

FIG. 3. (Color online) Schematic view of a suggested setup for experimental studies of long-lived neutron emitters by tracking their decay products in flight. The high-resolution neutron detector HRND is combined with a charged-particle detector for tracking the heavy fragments also with high angular resolution.

$\mathrm{MeV}$, half-lives are expected to cover the range from ps to sub- $\mu$ s. Therefore, the decay of such extremely short-lived radioactive ions can only be detected in flight directly after their production using the tracking technique. This technique, which was applied successfully for the observation of $2 p$ radioactivity of ${ }^{19} \mathrm{Mg}$ [5], appears to be most suitable here and will be discussed in the following. The decays of interest can be investigated by measuring angular correlations between each decay fragment stemming from the precursor decay in flight and the emitted neutron(s). The angular correlations provide information on energies and lifetimes of the parent nuclei. This tracking technique has the crucial advantage of providing data with high precision on exotic nuclei produced with very small rates, as compared to invariant-mass-method. We have performed Monte Carlo simulations of the decays of interest with the experimental setup sketched in Fig. 3. This setup comprises detectors for tracking the heavy daughter nuclei and the neutrons with high angular resolution. The neutron detector should be "thin" (to avoid possible cross-talk, because multiple scattering of a single neutron in a "thick" detector may mock a true $2 n / 4 n$ event) and, thus, will have only low efficiency for neutron detection. We have investigated two cases, the $2 n$ decay of ${ }^{26} \mathrm{O}$ and the $4 n$ decay of ${ }^{28} \mathrm{O}$. Though both parent

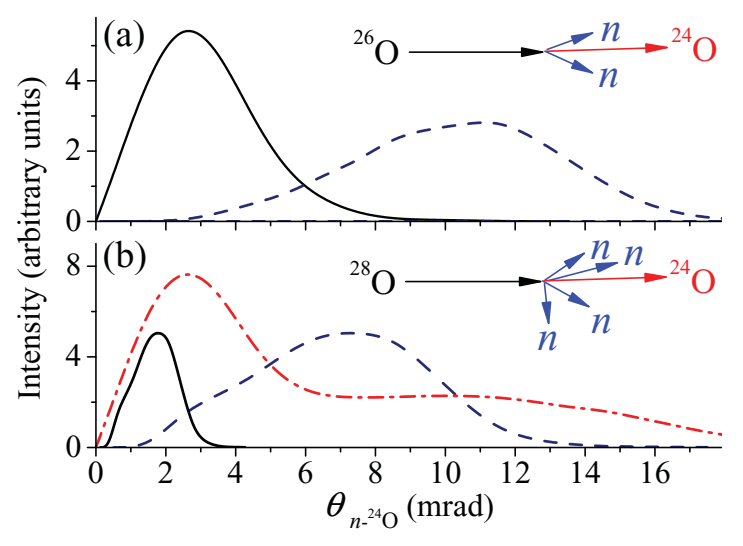

FIG. 4. (Color online) Monte Carlo simulations of the angular distributions between the heavy fragment and one of the neutrons for decay energies of $20 \mathrm{keV}$ (solid curves) and $300 \mathrm{keV}$ (dashed curves): (a) spectra for the true $2 n$ decay of ${ }^{26} \mathrm{O}$; (b) spectra for the true $4 n$ decay of ${ }^{28} \mathrm{O}$. The dash-dotted curve in (b) refers to a sequential $2 n-2 n$ decay of ${ }^{28} \mathrm{O}$ via the ${ }^{26} \mathrm{O}$ g.s. with $4 n$ - and $2 n$ - decay energies of 300 and $20 \mathrm{keV}$, respectively. 
nuclei are unknown, they are expected to be unbound and to decay to the ${ }^{24} \mathrm{O}$ ground state by $2 n / 4 n$ emission. We have assumed that the nuclei of interest are populated in secondary reactions with radioactive beams, e.g., in one-proton knock-out from ${ }^{27,29} \mathrm{~F}$ projectiles at energies of $\sim 500 \mathrm{~A} \mathrm{MeV}$, i.e., at typical GSI/FAIR energies. The simulated angular distributions of the decay products are shown in Fig. 4. It was demonstrated in true $2 p$ decays $[5,27]$ that such characteristic correlation patterns are indeed formed allowing one to identify the decay mechanism and to measure the decay energy.

As can be seen in Fig. 4(a), $2 n$ decay energies as low as very few $\mathrm{keV}$ can be reached provided that the experimental setup has an angular resolution of about $1 \mathrm{mrad}$. In the case of a true $4 n$ decay [see Fig. 4(b)], a single peak is formed in the angular correlation between the heavy fragment and one of the neutrons, which corresponds to uniform sharing of the decay energy among all neutrons. One should note that the correlation pattern appears to be sensitive to the decay mechanism, as the sequential $2 n-2 n$ decay via the ${ }^{26} \mathrm{O}$ g.s. provides a dramatically different correlation distribution. Similarly to the $2 n$ case, for the simulated $4 n$ decay, the necessary angular resolution of the neutron detectors should be about $1 \mathrm{mrad}$ as well. Existing detectors, like the present large-area neutron detector LAND at GSI [28], provide an angular resolution of $\approx 10 \mathrm{mrad}$, thus, new developments are needed.

In summary, the observation of neutron radioactivity in $s$-d shell nuclei seems unrealistic, but sufficiently long lifetimes may occur in decays of heavier ( $p-f$ shell) systems. We have estimated that lifetimes for true $2 n$ emission are much longer compared to the lifetimes of one-neutron emitters with the same energy. A similar effect is already known for true $2 p$ emission ( $2 p$ radioactivity) and understood theoretically. The trend toward longer lifetimes continues for true fournucleon emission, which should be strongly hindered as compared to true two-nucleon emission with the same decay energy. For that reason, the existence of $2 n$ and, especially, of $4 n$ radioactivity is plausible, since the energy windows corresponding to the radioactive timescale is estimated to be reasonably broad. The estimates show that the decay-energy conditions for true $4 p$ emitters are rather unlikely and that the $4 p$ decays are most likely to happen as a sequential $2 p-2 p$ emission. In contrast, the decay-energy conditions for true $4 n$ emission are likely fulfilled in ${ }^{7} \mathrm{H}$ and could be fulfilled in several other neutron-rich isotopes, e.g., ${ }^{18} \mathrm{Be}$ or ${ }^{28} \mathrm{O}$, which are in reach at modern radioactive beam facilities. The feasibility of an experimental search for long-lived true $2 n$ and $4 n$ emitters (i.e., with sufficiently small decay energy) by using a method of in-flight-decay and tracking of the decay products is demonstrated by our Monte Carlo simulations.

The work is supported by the Helmholtz International Center for FAIR within the LOEWE program by the State of Hessen, Germany. L.V.G. is supported by the FAIR-Russia Research Center and RFBR Grant No. 11-02-00657-a and the Russian Ministry of Industry and Science, Grant No. NSh-7235.2010.2.
[1] V. I. Goldansky, Nucl. Phys. 19, 482 (1960).

[2] M. Pfützner et al. Eur. Phys. J. A 14, 279 (2002).

[3] J. Giovinazzo et al. Phys. Rev. Lett. 89, 102501 (2002).

[4] B. Blank et al. Phys. Rev. Lett. 94, 232501 (2005).

[5] I. Mukha et al. Phys. Rev. Lett. 99, 182501 (2007).

[6] M. Pomorski et al. Phys. Rev. C 83, 061303(R) (2011).

[7] K. Miernik et al. Phys. Rev. Lett. 99, 192501 (2007).

[8] L. V. Grigorenko, I. G. Mukha, and M. V. Zhukov, Nucl. Phys. A 713, 372 (2003); 410, 401(E) (2004).

[9] A. S. Fomichev et al., Int. J. Mod. Phys. E 20, 1491 (2011).

[10] M. Thoennessen, Rep. Prog. Phys. 67, 1187 (2004).

[11] R. J. Charity et al. Phys. Rev. C 82, 041304(R) (2010).

[12] L. V. Grigorenko et al. Phys. Lett. B 677, 30 (2009).

[13] M. S. Golovkov et al. Phys. Rev. C 72, 064612 (2005).

[14] L. V. Grigorenko and M. V. Zhukov, Phys. Rev. C 76, 014008 (2007).

[15] L. V. Grigorenko and M. V. Zhukov, Phys. Rev. C 76, 014009 (2007).
[16] L. V. Grigorenko and M. V. Zhukov, Phys. Rev. C 77, 034611 (2008).

[17] A. Korsheninnikov et al. Phys. Lett. B 326, 31 (1994).

[18] M. S. Golovkov et al. Phys. Lett. B 672, 22 (2009).

[19] H. Johansson et al. Nucl. Phys. A 847, 66 (2010).

[20] D. R. Tilley, H. R. Weller, and G. M. Hale, Nucl. Phys. A 541, 1 (1992).

[21] A. Korsheninnikov et al. Phys. Rev. Lett. 90, 082501 (2003).

[22] M. Caamano et al. Phys. Rev. C 78, 044001 (2008).

[23] E. Nikolskii et al. Phys. Rev. C 81, 064606 (2010).

[24] M. S. Golovkov et al. Phys. Lett. B 588, 163 (2004).

[25] A. Volya, V. Zelevinsky, Phys. Rev. C 74, 064314 (2006)

[26] T. Otsuka, T. Suzuki, J. D. Holt, A. Schwenk, and Y. Akaishi, Phys. Rev. Lett. 105, 032501 (2010).

[27] I. Mukha et al. Phys. Rev. C 82, 054315 (2010).

[28] Th. Blaich et al. Nucl. Instr. Meth. A 314, 136 (1992). 